\title{
Cerebral organoids enter the fold
}

Two groups use cerebral organoids to model brain development and disease.

The human brain is unusually large and densely innervated, mainly because of an expanded cerebral cortex, the region associated with consciousness and higher brain function. The processes that give rise to this important structure and the diseases that affect it are of great interest to scientists like Arnold Kriegstein at the University of California, San Francisco and Rudolf Jaenisch at the Massachusetts Institute of Technology. In new work, both use cerebral organoids as a way to access early stages of brain development and to model malformations.

Organoids are stem-cell-derived, three-dimensional cultures that self organize to some extent and include multiple cell types and features of a particular organ. Kriegstein, with Marina Bershteyn, Tomas Nowakowski and their colleagues, generated cerebral organoids using induced pluripotent stem cells from individuals with Miller-Dieker syndrome (MDS), a genetic disease characterized by a 'smooth' or lissencephalic brain that lacks cortical folds (Bershteyn et al., 2017). They used the pioneering protocol of Yoshiki Sasai, in which dissociated stem cells reaggregate in suspension and then differentiate into cortical organoids in the presence of growth factor cues.

Their work successfully recapitulated key features and stages of lissencephaly, including a combination of cell death and division defects in neuroepithelial progenitors, as well as cellautonomous neuronal migration defects in MDS compared to wild type organoids. Cerebral organoids do have limitations, however. In particular, they currently only model fetal development in the first two trimesters, making them less suited for neurodegeneration and other diseases with later onset. "I think it's premature to look at anything that relates to a cortical circuit in an organoid," adds Kriegstein. And missing cell types, including immune cells, can make it tricky to interpret disease phenotypes.

Jaenisch and his team were driven by the question of why rodent brains are smooth whereas

\section{GENOMICS}

\section{ANOTHER PLAYER FOR RNA-GUIDED RNA CLEAVAGE}

\section{Cas13b relies on activator and repressor proteins to regulate RNA cleavage.}

The CRISPR-Cas9 system has taken the world of genome editing by storm, but Cas 9 was just a forerunner of many other RNA-guided nucleases. While many people will be familiar with the basics of Cas9-mediated DNA editing -its requirement for a guide RNA complementary to its DNA target and a protospacer adjacent motif (PAM) next to the target site-the diversity of members in the class II CRISPR-Cas system may take some by surprise.

In a recent review Eugene Koonin from the NIH presented an updated phylogenetic tree of class II Cas proteins, split into type II such as Cas9, type V such as Cpf1 and the RNA-targeting type VI such as C2c2 (Shmakov et al. 2017); but since its publication, several additional family members had been discovered, such as DNA-targeting CasX and CasY (Burstein et al. 2017). Another recently added member is Cas13b, discovered in a collaboration between Koonin and Feng Zhang, who is at the Broad Institute.

Zhang says he was mainly driven by curiosity to see which other CRISPR systems were out there. "Can we harness them to do something cool?" he asked. To embark on this project three graduate students in the Zhang lab pooled their expertise; Aaron Smargon led the computational effort, David Cox oversaw bacterial genetics, and Neena Pyzocha directed the biochemical work (Smargon et al. 2017).

The CRISPR locus is conserved in bacteria, and previous efforts to find new Cas proteins have searched for the presence of Cas1 and Cas2, enzymes that incorporate new DNA into CRISPR repeats. But Zhang found this approach too restrictive as "there could be other systems that don't contain Cas1 or Cas2," he explained. Thus, Smargon set up a pipeline to look for conserved but uncharacterized large proteins near CRISPR repeat sequences and for the absence of cas1 and cas2 genes. This led to the discovery of Cas13b, a member of subtype VI-B, with two active domains that suggest the protein cleaves RNA. 


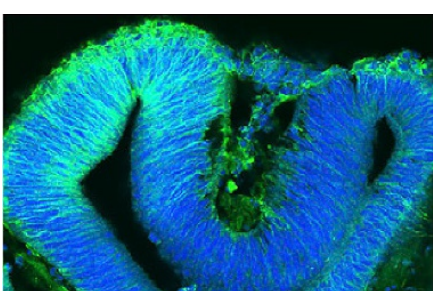

Cerebral organoids are tractable models of early brain development. et al., 2017. Reproduced with permission from $\mathrm{Li}$

primate brains are folded (Li et al., 2017). They used a robust protocol, developed by collaborators Madeline Lancaster and Juergen Knoblich, which also starts with free-floating stem cell aggregates that are then cultured in extracellular matrix. In matrix, multiple brain regions can spontaneously develop within a single organoid. The team discovered that genetic lesions in the anti-proliferative gene PTEN cause neural progenitor populations to expand and delay neuronal differentiation, but only lead to folding in human and not similarly cultured rodent cortical organoids. The exact mechanism of folding in human embryos is still somewhat unclear, however, as it occurs later and involves a different cortical structure.

Jaenisch also sees directions for improving organoids. For example, neurons begin to die once organoids reach a certain size. "One would probably like to bioengineer something better," he says, referring to a circulation system that would allow larger and longer-lived organoids. Variability is also an issue; for now, large numbers of organoids must be analyzed until protocols produce more consistent results.

Despite some limitations, organoids provide powerful and tractable models of early brain development and disease. The Jaenisch team found that infection with Zika virus, which has been associated with microcephaly, disturbed cortical growth and folding, and both groups are developing further studies of viral infection using organoids.

\section{Tal Nawy}

\section{RESEARCH PAPERS}

Bershteyn, M. et al. Human iPSC-derived cerebral organoids model cellular features of lissencephaly and reveal prolonged mitosis of outer radial glia. Cell Stem Cell http://dx.doi.org/10.1016/j.stem.2016.12.007 (2017). Li Y., et al. Induction of expansion and folding in human cerebral organoids. Cell Stem Cell http://dx.doi. org/10.1016/j.stem.2016.11.017 (2017).

The first question was whether this protein mediated CRISPR-based interference in bacteria. Smargon set up a screen in Escherichia coli targeting Cas13b to 45 essential genes with 54,600 guide RNAs. He saw a depletion of those gRNAs over nontargeting controls indicating that Cas $13 \mathrm{~b}$ activity led to cell death. Pyzocha purified the Cas13b protein and showed in in vitro screens that it indeed cleaves single-stranded RNA.

These results allowed them to draw first conclusions about rules governing the targeting. Unlike other Cas proteins with a unique PAM either upstream of downstream of the gRNA binding site, Cas13b has a protospacer flanking sequence (PSF) on either side of the gRNA. The exact interaction between the Cas13b-gRNA complex and PSFs will be the subject of further studies.

Another unique feature of Cas13b is the interaction with its accessory proteins Csx27 and Csx28, two small proteins encoded within the CRISPR locus. When the researchers infected $E$. coli with the RNA phage MS2 and targeted Cas13b against MS2, they saw that Csx27 weakened RNA cleavage, whereas Csx28 enhanced it.

The precise role of these proteins is not yet clear, but Zhang sees the need for additional safeguards in a system that targets RNA. As the in vitro screen showed, Cas13b-like other RNA-guided RNA nucleases-displays collateral activity. Once the nuclease is activated by cleaving its specific target, it remains active and cleaves any RNA.

Zhang is confident that by understanding more about the structure of Cas $13 \mathrm{~b}$ and its mode of action researchers will be able to control nonspecific cleavage and possibly use it for targeted RNA interference in mammalian cells down the line.

Nicole Rusk

RESEARCH PAPERS

Shmakov, S. et al. Diversity and evolution of class 2 CRISPR-Cas systems. Nat. Rev. Microbiol. http://dx.doi. org/10.1038/nrmicro.2016.184 (2017).

Burstein, D. et al. New CRISPR-Cas systems from uncultivated microbes. Nature http://dx.doi.org/10.1038/ nature21059 (2016).

Smargon, A.A. et al. Cas13b is a type VI-B CRISPR associated RNA-guided RNase differentially regulated by Csx27 and Csx28. Mol. Cell. http://dx.doi.org/10.1016/j.molcel.2016.12.023 (2017). 